\title{
3D spectroscopy of dwarf elliptical galaxies
}

\author{
Olga K. Sil'chenko ${ }^{1, a}$ \\ Sternberg Astronomical Institute, Moscow, Russia
}

\begin{abstract}
I present some results of 3D spectroscopy for a small sample of dwarf elliptical galaxies, mostly members of small groups. The galaxies under consideration have a typical absolute magnitude of $-18(B)$, and at the Kormendy's relation they settle within a transition zone between the main cloud of giant ellipticals and the sequence of diffuse ellipticals. By measuring Lick indices and investigating radial profiles of the SSP-equivalent ages and metallicities of the stellar populations in their central parts, I have found evolutionary distinct cores in all of them. Typically, the ages of these cores are 2-4 Gyr, and the metallicities are higher than the solar one. Outside the cores, the stellar populations are always old, $T>12 \mathrm{Gyr}$, and the metallicities are subsolar. This finding implies that the well-known correlation between the stellar age and the total mass (luminosity) of field ellipticals [12]3 may be in fact a direct consequence of a larger contribution of nuclear starbursts into the integrated stellar population in dwarfs with respect to giants, and does not relate to 'downsizing'.
\end{abstract}

\section{Introduction}

The results of panoramic spectroscopy and spatially resolved parameters of the stellar populations (ages and metallicities) for 6 dwarf elliptical galaxies are presented in this contribution. All of the galaxies have the blue absolute magnitude of about -18 . Their positions on the absolute magnitude-surface brightness and absolute magnitude-effective radius diagrams classify them as the faint extremity of the ordinary elliptical galaxies family, though their effective radii are rather small, and at the Kormendy's diagram they are located somewhat between the ordinary ellipticals and diffuse elliptical galaxies. All 6 targets are members of loose galaxy groups; but 3 of them look rather isolated, and 3 of them are projected very close to the bright early-type galaxies. We cannot characterize them however as interacting galaxies because the relative line-of-sight velocities are as high as 300-700 $\mathrm{km} / \mathrm{s}$.

\section{Observations and data used}

I present here some integral-field spectral data obtained with the Multi-Pupil Fiber Spectrograph (MPFS) of the Russian $6 \mathrm{~m}$ telescope and the SAURON data retrieved from the public ING Archive of the UK Astronomy Data Center. For the MPFS description, see 4], and for the SAURON description, see [5]. Briefly, the former spectrograph gives the field of view of $16^{\prime \prime} \times$ $16^{\prime \prime}$ and the spectral range of $1500 \AA$ under the spectral resolution of $3 \AA$; the blank sky to subtract from

\footnotetext{
${ }^{a}$ e-mail: olga@sai.msu.su
}

the galaxy spectra is taken at 4 arcmin from the target. The SAURON possesses the field of view of $41^{\prime \prime} \times$ $33^{\prime \prime}$ and the spectral range of $550 \AA$ under the spectral resolution of $4 \AA$; the blank sky is exposed at 1.7 arcmin from the center of the field of view. The spaxel is about 1 arcsec in both spectrograph. NGC 3605 has been observed with the MPFS in April 2005. All six dwarfs were also exposed with the SAURON in 2007 2008 in the frame of the project ATLAS-3D. I have calculated the Lick indices $\mathrm{H} \beta$, Mgb, and Fe 5270 for every spaxel. The radial profiles of the Lick indices obtained for NGC 3605 with the MPFS and with the SAURON agree well.

\section{Results and Discussion}

Figure1demonstrates the way to determine the luminosityweighted (SSP-equivalent) age and metallicities of the stellar populations in the nuclei and in the main bodies taken outside $R=3^{\prime \prime}$. I confront the measured Lick indices, $\mathrm{H} \beta$ and the combined magnesium-iron index $[\mathrm{MgFe} 52]$, to the population-synthesis models for old SSP by 6]. The $\mathrm{H} \beta$ of the nucleus in NGC 3522 has been corrected for the weak emission, $\Delta \mathrm{H} \beta=$ $0.6 E W([\mathrm{OIII}] \lambda 5007)$. Table 1 presents the results of this analysis. In ALL elliptical dwarfs studied here the nuclei are chemically and evolutionarily decoupled from the main bodies of the galaxies, the nuclei being younger and more metal-rich. The metallicity differences between the nuclei and the main bodies reach 3-6 times (0.5 - $0.8 \mathrm{dex})$ while typical enrichments for the chemically decoupled nuclei in giant galaxies are known to be around 2 times $(0.3$ dex $)$ [7]. Evidently, 
Table 1. The ages and metallicities for the cores and main bodies

\begin{tabular}{|l|cc|cc|}
\hline NGC/UGC & \multicolumn{2}{|c|}{ NUCLEUS } & \multicolumn{2}{|c|}{ MAIN BODY $\left(R>3^{\prime \prime}\right)$} \\
& Age, Gyr & {$[\mathrm{Z} / \mathrm{H}]$} & Age, Gyr & {$[\mathrm{Z} / \mathrm{H}]$} \\
\hline $\mathrm{N} 3522$ & $3 \pm 1$ & +0.2 & $8 \pm 0.5$ & $<-0.3$ \\
$\mathrm{~N} 3605$ & $4 \pm 1$ & +0.3 & $7 \pm 1$ & -0.2 \\
N3641 & $7 \pm 2$ & +0.2 & $12 \pm 3$ & -0.3 \\
N4283 & $3 \pm 0.5$ & +0.7 & $13 \pm 1$ & -0.1 \\
N5500 & $3 \pm 1$ & +0.4 & $>12$ & $<-0.3$ \\
U3960 & $10 \pm 2$ & -0.2 & $>12$ & $<-0.3$ \\
\hline
\end{tabular}

the stellar nuclei have been formed dissipatively, in intense secondary star formation bursts. The main bodies of all the dwarfs except NGC 3605 are older than 8 Gyr; in NGC 3605 the nuclear starburst has been perhaps more extensive than in other galaxies that is also implied by asymmetric large-scale distribution of the blue colour in this galaxy. We may suggest that the young SSP-equivalent age found by e.g. 2 for nearby dwarf early-type galaxies, $\langle T\rangle=3.6 \mathrm{Gyr}$, relates precisely to the nuclei, not to the whole galaxies. Then the correlation between the mass of a galaxy (stellar velocity dispersion) and the age of its stellar population found more than once among nearby elliptical galaxies, e.g. by 13 , and others, which is usually treated as a manifestation of 'downsizing', is in fact an artifact caused by the use of the data of aperture spectroscopy centered onto the nuclei. Minor mergers are frequent events among all types of galaxies, and they have to provoke nuclear starbursts if the gas is involved; but in a dwarf galaxy the contribution of the products of nuclear starburst into the total luminosity of the central part of the galaxy must be more impressive than in a giant one. So their centers look younger than the centers of giant ellipticals. If we take the main bodies as representative for the whole galaxies, the dwarfs would appear as old as the giants.

\section{Acknowledgements}

This research is based on data obtained from the Isaak Newton Group Archive which is maintained as part of the CASU Astronomical Data Centre at the Institute of Astronomy, Cambridge, and also on the data obtained at the Russian $6-\mathrm{m}$ telescope. The $6 \mathrm{~m}$ telescope of the Special Astrophysical Observatory is operated under the financial support of the Ministry of Science and Education of Russian Federation (registration number 01-43).

\section{References}

1. S. C. Trager, S. M. Faber, G. Worthey, J. J. Gonzalez, 120, 165 (2000)

2. N. Caldwell, J. A. Rose, K. D. Concannon, AJ 125, $2891(2003)$

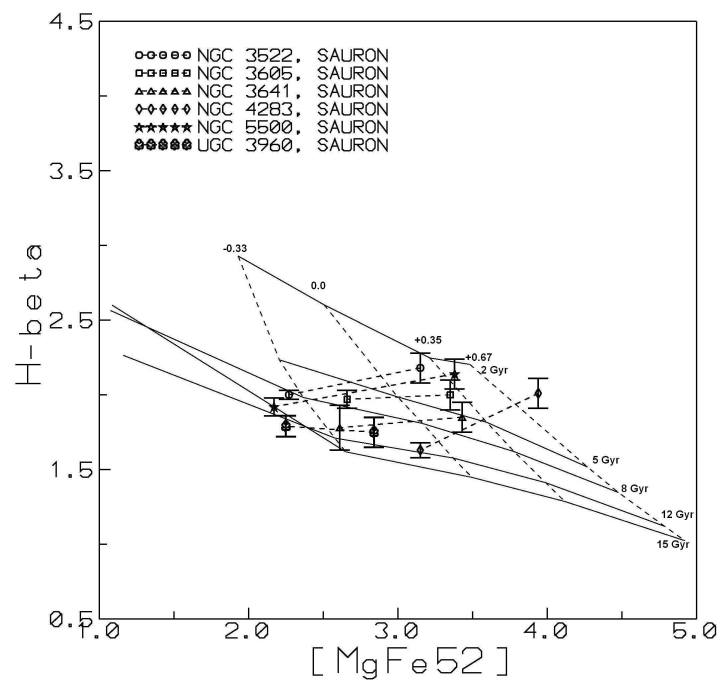

Fig. 1. The index-index diagnostic diagram to determine luminosity-weighted ages and metallicities of the stellar populations. Every pair of signs connected by dashed straight lines relates to one galaxy; every right reper corresponds to the nucleus and every left reper is the averaged off-nuclear data taken at $R>3^{\prime \prime}$. The labeled solid and dashed lines are constant-age and constant-metallicity model sequences by Thomas et al. (2003).

3. J. H. Howell, AJ 130, 2065 (2005)

4. V. L.Afanasiev, S. N.Dodonov, A. V.Moiseev, In : Stellar dynamics: from classic to modern/Eds. Osipkov L.P. and Nikiforov I.I. (St. Petersburg Univ. Press, St. Petersburg, 2001) 103

5. R. Bacon, Y. Copin, G. Monnet, B. W. Miller, J.

R. Allington-Smith, et al., MNRAS 326, 23 (2001)

6. D. Thomas, C. Maraston, R. Bender, MNRAS 339, 897 (2003)

7. O.K. Sil'chenko, ApJ 641, 229 (2006) 